\title{
Managing bottleneck resources in production networks
}

\author{
Hans-Peter Wiendahl, Katja Windt, Jens Lopitzsch and Michael Schneider \\ Institute of Production Systems and Logistics, University of Hannover, Callinstraße 36, D. \\ 30167 Hannover, Germany. Email: wiendahl@ifa.uni-hannover.de
}

\begin{abstract}
The subcontracting process within production networks is an essential measure to realize high logistics performance in terms of high delivery reliability and short lead times. Flexible capacities still prove to be too expensive especially for small and medium-sized suppliers. In this situation, production networks offer the possibility of relying on the capacities of other network partners if capacity shortages arise. The essential prerequisites to successfully execute the subcontracting function within a production network are the identification and elimination of bottleneck resources in the production process. This paper presents a methodology which allows the identification and classification of bottleneck work centres according to different bottleneck criteria. By use of this bottleneck analysis for the production process adequate measures for eliminating present bottlenecks, and for avoiding future ones, can be derived.
\end{abstract}

Keywords

Logistics performance, bottleneck analysis, bottleneck classification, production network, subcontracting, Logistic Operating Curves.

\section{INTRODUCTION}

Intensifying cost pressures and globalization efforts force manufacturers to revise their business strategies and production concepts. As customers' decision to buy a product depends on short and reliable delivery times, many companies see the necessity of reducing throughput times and of stabilizing processes. Investigations of effective methods for pursuing these objectives prove that, first of all, the overload of the production system by uncontrolled order release has to be avoided. Overload leads directly to increasing queues. By this, throughput times are prolonged, the deviation of throughput time increases and the delivery reliability decreases. So, the amount of work released must be adjusted to actual capacities. That is why, there is an essential need for flexible capacities in case of fluctuating demands. Especially small and medium-sized businesses have severe difficulties in meeting the fluctuations of their customers' demands. Partly, these problems can be solved with flexible working times and jumper concepts. However, due to restrictions concerning legal working times and awkward union contracts, mostly, 
the scope for time adjustments is limited. In this situation production networks offer an extensive solution (Grandori et al., 1995; Nassimbeni, 1998). Production networks incorporate numerous cooperating companies over a limited time span. In case of an overload situation of one network partner, entire customer orders or even single processes can be subcontracted to partner companies disposing of available production capacity. The final responsibility for the product is passed on to the production network rather than single companies. Nevertheless, these orders or process steps must be accurately identified in order to keep utilization rates at a high level. The approach described supports the process of sensible and effective subcontracting in production networks based on the four logistic objectives (Wiendahl, 1995). By this method network partners will get reliable hints for their decisions about subcontracting.

\section{THE PRODUCTION NETWORK - AN APPROACH FOR COLLABORATIVE PRODUCTION}

\section{Trends and history}

During the last few years changes in structural organization have turned away from the original strong hierarchy to lean management and a further step towards the formation of small, almost independent factory units in the form of profit and/or cost centres. This development was further supported by the parallel introduction of team and group working. The companies' internal focus on smaller units in the area of the structural organization was overlapped by different activities to change the process organization. Processes were optimized and accelerated. At a first attempt, resources were adjusted to new conditions. In the follow up, many enterprises started a phase of restructuring (business-process reengineering) in which existing organization forms and processes were segmented according to technological or product-related criteria. Then, the product and the process operation were redesigned in a fourth stage (see Figure 1).

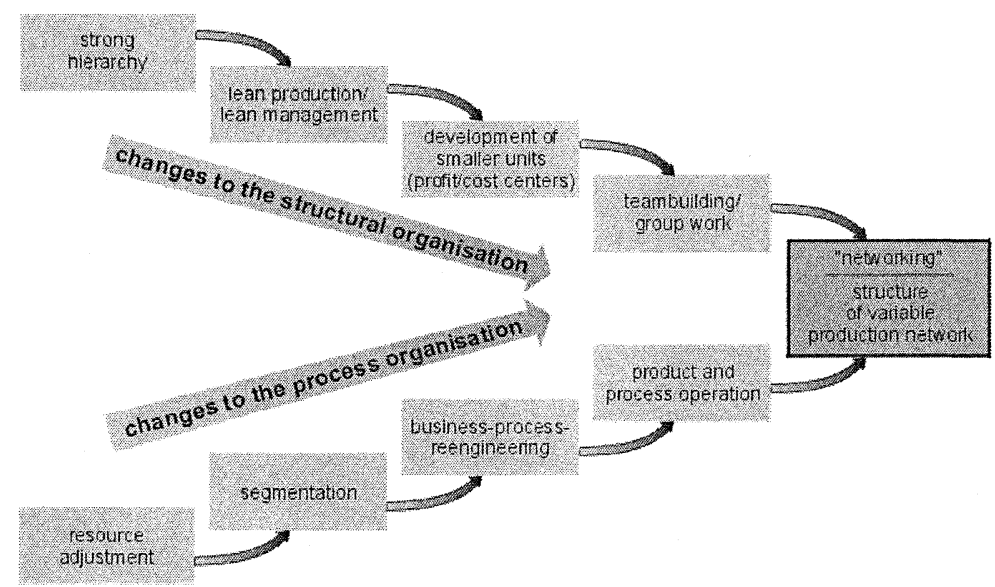

Figure 1 - Phases of restructuring of production enterprises 
This step-wise optimization of structural organization and process organization shows the general trend in many companies towards increased cooperation (Starr, 1991). In the past, cooperation in the areas of research and development has already proved its worth. Nowadays, links between procurement, production and distribution departments are being increasingly built up and used. That leads in the sense of vertical integration of customer-supplier relations to logistic supply chains. The outcome of the further horizontal integration are stable network arrangements. The highest level of cooperation with changing partners and flexible network states can then be identified as the production network. It is characterized by intensive communication between the participating companies even though they might represent the same level of value adding. That means, that in a production network the suppliers of a company have to communicate with each other as well as with the company itself, leading to an intensive flow of information between all participants in a production network. This intensive flow of data should help the networking partners to plan more accurately and to adjust their capacities more effectively since information is provided both earlier and in more detail. In order to support this process of data processing new methods and concepts are required (Semich, 1994). Thus, production networks facilitate the avoidance of large deviations of demand along the value added chain as described by (Forrester, 1961) and as demanded by aiming high delivery reliability.

\section{An Example}

According to its basic definition, a production network represents a dynamic, reconfigurable connection of numerous companies over time. Figure 2 shows as an example the networking of a number of companies to produce micro-electronic components. For visualization purposes a universal process chain model was used (Kuhn, 1995) leading to the networked process chain plan presented. This plan proves that a production network can be fully described by the three forms of process chain elements: Producing/ Testing (P), Transporting (T) and Storage/ Supply (S). In this example, the companies $\mathrm{P} 1$ and $\mathrm{P} 2$ supply the raw material for production of micro-electronic components, in this case a wafer. This is followed by a mechanical operation (companies P3 and P4) in which the production photolithography, loading and removal connect up from differing spatial classes. Parallel to this, the companies P12 and P13 work in an identical process chain. They are in the position to carry out the same production already described. There follows a common, cost-intensive testing process. Then, wafers are separated by the companies P8 and P14. The production process structure and connection technology integrates all necessary components in a single casing. The store $\mathrm{S} 2$ holds all the finished wafers for future work (e.g. for production of an acceleration sensor). The actual production process is finished at this point and the final product can be handed to the distributor.

Characteristically, redundancies are a fundamental element in production networks (Wiendahl et al., 1998; Wiendahl et al., 1998a). They are planned deliberately. This means, that more than one process or partner produces the same product or provides the same service. In Figure 2, for example, the processes P5 and P12 or the processes P6 and P13 are redundant. Both processes have an identical 
input and output. Redundancy increases the flexibility of the network and the assurance of supply for the customer.

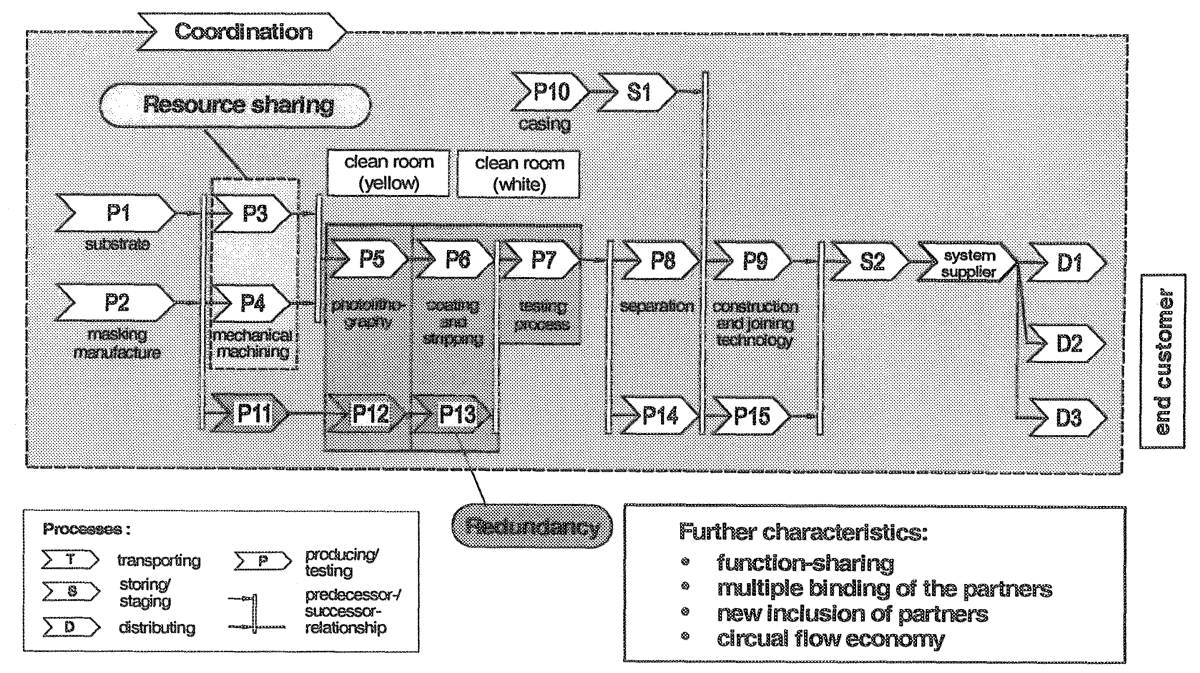

Figure 2 - Variable production network for manufacturing of microelectronic parts

A further typical attribute of production networks are shared resources to avoid bottlenecks and to reduce high capital expenditures on work centres. Each networked process step disposes of resource pools, that consists of all necessary resources to execute this process. Resources sharing means, that two or more companies share lean resources dependent on situation and demand and with alternating rights of first choice. In Figure 2, the companies executing P3 and P4 share resources (operation devices, personnel, material, media, etc.) as well as $\mathrm{P} 9$ and P15. The sharing process can be planned on a long-term basis or it is used for spontaneous avoidance of bottlenecks.

\section{Challenges on enterprises in production networks}

Only cooperations based on equal rights of each networking partner can take the full benefit from the production network. Factors like frankness, flexibility, sharing of resources and functions as well as the ability to multi-cooperation describe more details of the successful partner.

Accurate information is an important prerequisite for the use of other partners' resources and for the allocation of orders to particular network partners according to their actual capability and performance. A second prerequisite is the confidence between the partners involved. The information shared by the participants, e. g. the actual capacity utilization of particular work systems, has so far only been handled internally inmost companies. In networks, this data is now available to external partners. This leads to a new stage of confidence. Hence the net partners have to agree in advance which data should be exchanged and how this data may be 
handled. Agreements have to be reached which guarantee that no confidential information is passed on to competitors. Alternatively, the data that should be exchanged can be filtered depending on the need of information and the intensity of the integration into the net. In this case, only pre-agreed data is exchanged and the company has complete control over its own data. However, there are further challenges especially on small and medium sized enterprises.

\section{Raised efforts of coordination}

Cooperation always means processes of tuning, arrangement and consideration. This is also referred to as the exploring and determining phase of possible cooperation, the phase of task definition, the operation phase as well as the phase of terminating and detaching cooperations.

\section{Use of modern ICT's}

New developments on the market of information and communication technologies have to be transferred to production networks and integrated in existing systems. Advantages of integrative information systems must be used: transparent information itself, more simple and efficient processes, less waste of information by undefined interfaces between different network partners. There is a need for algorithms that allow this straight and immediate data exchange without further efforts on formatting information and switching information systems. But, not only the process of data or information exchange has to be improved. It is also necessary to provide information to the network partners in a much wider complexity. Therefore, attitudes concerning data protection and separation of confident in-house data from public data have to be fundamentally revised.

\section{Demand on new methods for production planing and control}

Investigating operating production networks proves that the network potentials are neither yet exploited with today's information and communication technologies nor they are with today's planing, control, and visualization systems (Spur, 1996). As resources are provided to the network, on the one hand, new instruments to observe, inform and derive sanctions are needed. On the other hand, networking requires special methods for planning and controlling (Inger et al., 1995; Kernler, 1995). These must especially enable the sharing of resources and the control of redundant processes. Actual systems contain these functions only very partly (Dombrowski, 1995). Furthermore, an all-time transparency of the production state is required to allow sensible subcontracting decisions. Accurate production control is fundamentally based on continuous supervision and on well-defined control mechanisms.

\section{Efficient resource sharing}

To use potentials of capacity flexibility in production networks, scheduling and resource planing must be adjusted. The coordination of production orders within a network asks for far reaching transparency, i.e. the possibility for order tracing within the partners processes. However, one of the most important reasons for 
networking is to avoid overload and underload situations by sharing resources among the network partners. The efficient resource sharing depends directly on knowledge about bottlenecks. That is why relevant bottlenecks must be detected and unplanned loading situations of work centres must be determined or even foreseen.

\section{METHODOLOGY FOR IDENTIFYING AND CLASSIFYING BOTTLENECK RESOURCES}

\section{Bottleneck definition}

A bottleneck can generally be defined as an imbalance between supply and demand. Two views can be distinguished: the resource and the order-oriented view. Resource-oriented bottlenecks refer to e.g. work centres or personnel. Different types of orders, parts and raw materials represent measures of order-oriented bottlenecks. To determine a bottleneck, the deviation between planned and actual data has to be analyzed (Figure 3).

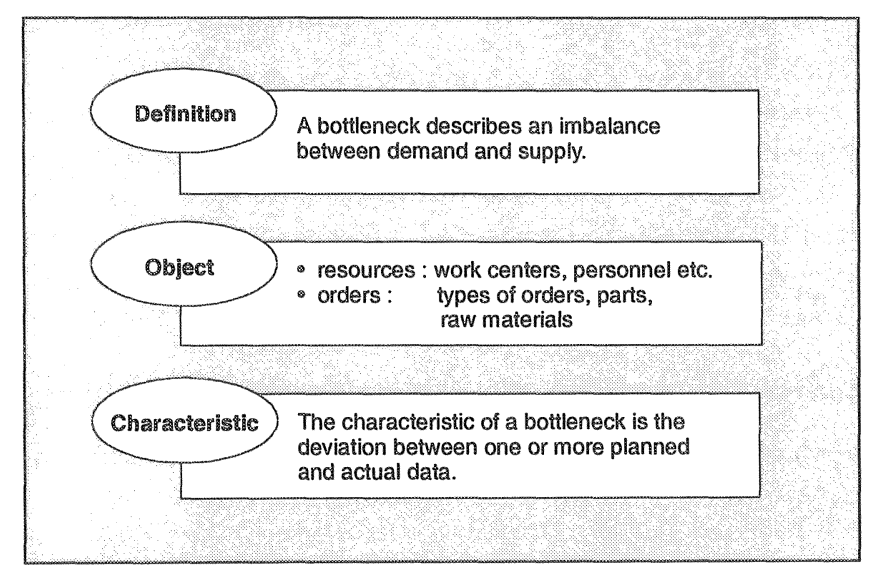

Figure 3 - Definition of a bottleneck

In practice, a production bottleneck is often defined as the work centre which limits throughput. However, enterprises aim at more than supporting just one objective. Normally, a combination of several logistic key factors is focussed. It is possible to divide as well these objectives according to their resource and order orientation. The order-related objectives are minimal due date deviation, short delivery times and high throughput. The objectives high utilization rate and low inventory level belong to the resource-oriented view. Furthermore, high efficiency (minimal costs with a given performance level) is obviously pursued. According to recent research studies (Windt, 2001) all these objectives can be supported by defining and controlling bottleneck types in a similar way.

This paper focuses on targets determining delivery time and delivery reliability in order to improve the logistic performance of the production investigated. Identifying the corresponding bottlenecks, the capital tied-up is indirectly influenced 
by aiming at low throughput times enabled by low WIP level. Thus the consideration of costs in terms of capital tied-up is indirectly included in the methodology. Whereas aspects as costing and pricing in the classical sense of performance measures are excluded from this methodology and may have to investigated in future research. Nevertheless recent basic research results show that the consideration of costs in direct combination with the logistic objectives can be possible (Kerner, 2001).

\section{Classifying bottlenecks}

The structure of the resource-and order-oriented bottleneck methodology is shown in Figure 4 Windt, 2001). The actual values for the identification of a bottleneck regarding the relevant objectives are calculated for each work centre. The calculations are carried out on the basis of shop floor feedback data. This data can be drawn from the logistic monitoring and controlling system of the company. It should provide detailed information about the status of orders and the availability of resources. The comparison of the planned and the actual values describes the deviation of the target Figures in terms of the bottleneck definition. Work centres which show the highest deviation represent so-called resource bottlenecks. The types of bottlenecks are being named respectively to their target criteria:

* due-date-determining bottleneck,

- throughput-determining bottleneck,

m throughput-time-determining bottleneck,

- utilization-determining bottleneck,

- WIP-determining bottleneck and

a capital-tied-up-determining bottleneck.

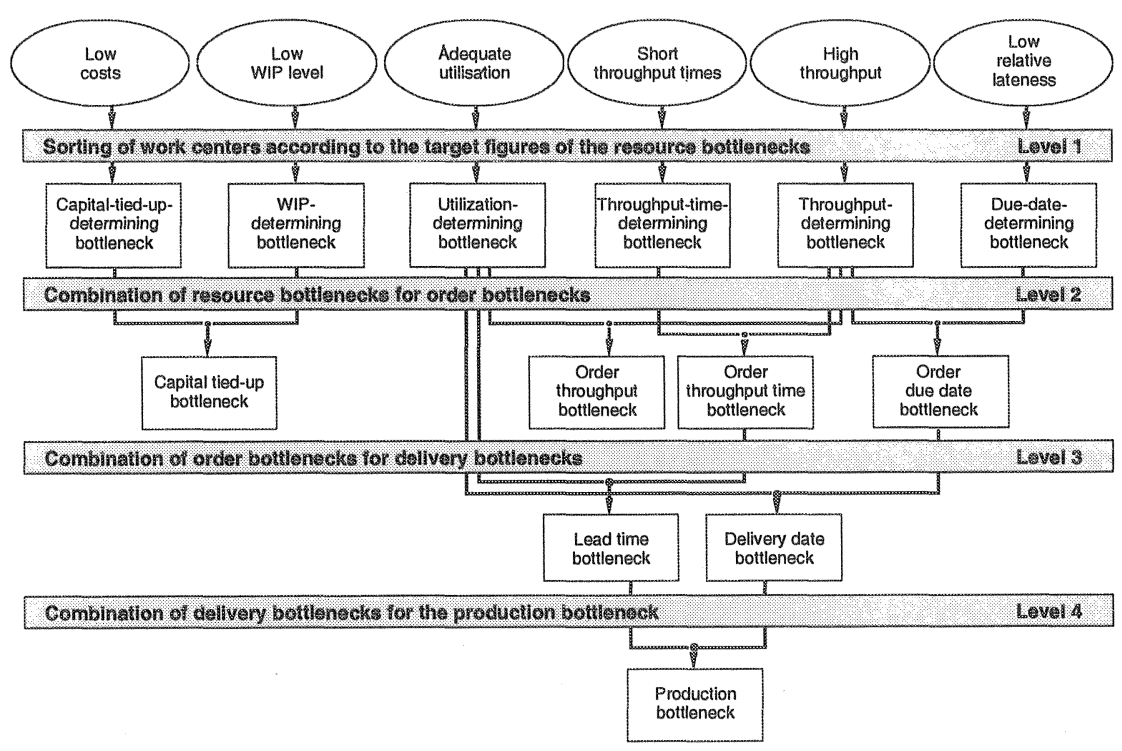

Figure 4 - Resource-and Order-oriented types of bottlenecks in production management 
The capital tied-up bottleneck represents a combination of the bottleneck criteria of low costs and low WIP level. However, as the consideration of costs is not going to be particularized in the presented bottleneck methodology the capital tied-up bottleneck is not going to be analyzed any further in this paper.

The bottlenecks of the $3^{\text {rd }}$ level can be identified by taking into consideration WIP-induced utilization. Thus it is possible to determine those work centres which can be affected in accordance with the bottleneck target criteria when the mean WIP is changed. These bottlenecks are a result of the combination of order bottlenecks and resource bottlenecks. They are delivery bottlenecks: delivery date bottleneck and lead time bottleneck.

Although the two types of delivery bottlenecks have been defined the work centre which has the strongest effect on the achievement of the bottleneck objectives has not been determined yet. This production bottleneck belongs to the $4^{\text {th }}$ level. It can be identified with the results of the combination of the two delivery bottlenecks. The $4^{\text {th }}$ level is the highest hierarchy level in the bottleneck methodology presented.

This paper shows the calculation of the order bottleneck, the delivery bottleneck as well as the production bottleneck in principle. The single steps are explained on the basis of real feedback data.

\section{Identifying resource bottlenecks and order bottlenecks}

The first step of the bottleneck methodology is the determination of the resource bottlenecks (bottlenecks of the $1^{\text {st }}$ level). Regarding for example the objective of short throughput time, the work centres are sorted in a hit list according to their mean throughput time. That work centre which achieves the longest mean throughput time is the throughput time determining bottleneck. For the subsequent calculation of the order bottleneck the results of two resource bottleneck analyses are combined in order to assess a combination of bottleneck target Figures. This is done by multiplying the chosen bottleneck Figures. As a result the work centres can be sorted due to their relative proportion of the combined bottleneck target Figures. In the following the equations for the calculation of the order throughput time bottleneck, the order due date bottleneck and the order throughput time bottleneck are going to be defined (Windt, 2001).

\section{Order throughput time bottleneck (bottleneck of the $2^{\text {nd }}$ levell)}

The increasing importance of short throughput times for manufacturing enterprises emphasizes the relevance of the order throughput time bottleneck. For its calculation the results of the throughput determining bottleneck analysis are combined with the values of the throughput time bottleneck analysis. The sum of all order throughput times TOT equals the sum of all operation throughput times TTP and the sum of all products of mean throughput time of a work centre $T T P_{m}$ and number of operations $n$ that have been processed (Nyhuis and Wiendahl, 1999)

$$
\sum_{k=1}^{s} T O T_{k}=\sum_{j=1}^{N W C} \sum_{i=1}^{n} \operatorname{TTP}_{i, j}=\sum_{j=1}^{N W C}\left(\operatorname{TTP}_{m} * n\right)_{j}
$$


where:

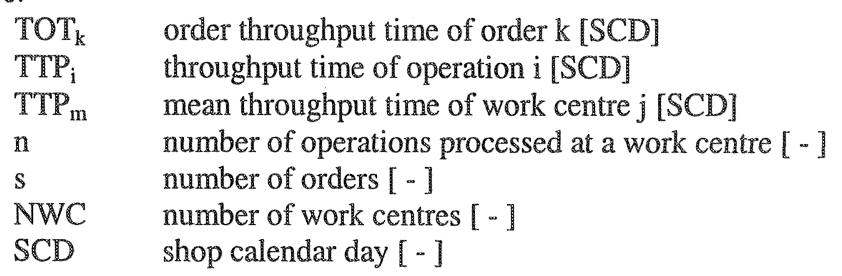

The number of operations that have been processed represents the throughput of orders of each work centre. The product of mean throughput time and number of orders that have been processed describe directly every work centres share of the order throughput time. The relative share of each work centre $T O T_{r e l}$ in terms of percent is:

$$
\operatorname{TOT}_{r e l, j}=\frac{\left(\operatorname{TTP}_{m} * n\right)_{j}}{\sum_{j=1}^{N W S}\left(\operatorname{TTP}_{m} * n\right)_{j}} * 100
$$

where:

$\mathrm{TOT}_{\text {rel,j }} \quad$ relative share of order throughput time of work centre j [\%]

The bottleneck analysis of the $1^{\text {st }}$ level does not offer this type of analysis. Thus those work centres can be located which endanger the bottleneck objectives of high throughput of orders as well as short throughput times.

\section{Order due date bottleneck (bottleneck of the $2^{\text {ndd }}$ level)}

Equation 1 can be adapted to describe the order delivery date bottleneck. Consequently the sum of the relative order lateness $L O$ equals the sum of the relative lateness of all operations LOP. This is equal to the sum of the products of mean relative lateness $L O P_{m}$ and the number of operations $n$ that have been processed:

$$
\sum_{k=1}^{s} L O_{k}=\sum_{j=1}^{N W C} \sum_{i=1}^{n} L O P_{i, j}=\sum_{j=1}^{N W C}\left(\operatorname{LOP}_{m} * n\right)_{j}
$$

where:

$\mathrm{LO}_{\mathrm{k}} \quad$ relative lateness of order $k$ [SCD]

$\mathrm{LOP}_{\mathrm{i}} \quad$ relative lateness of operation $\mathrm{i}$ [SCD]

$\mathrm{LOP}_{\mathrm{m}} \quad$ mean relative lateness of work centre $\mathrm{j}$ [SCD]

The relative share of each work centre $L O_{r e l}$ in terms of percent is:

$$
L O_{r e l, j}=\frac{\left|\operatorname{LOP}_{m} * n\right|_{j}}{\sum_{j=1}^{N W S}\left|\operatorname{LOP}_{m} * n\right|_{j}} * 100
$$

where: 
$\mathrm{LO}_{\mathrm{rel}, \mathrm{j}} \quad$ : relative share of order lateness of work centre $j[\%]$

\section{Order throughput bottleneck (bottleneck of the $2^{\text {nd }}$ level)}

The main intention of this bottleneck analysis is to find that work centre which limits total output of production. This work centre is characterized by a high number operations and high utilization due to a high WIP level. In consequence, the order throughput bottleneck can be identified by calculating the product of the number of operations $n$ and the relative WIP WIP rel. A work centres relative share $O T P_{r e l}$ is:

$$
\operatorname{OTP}_{r e l, j}=\frac{\left(W I P_{r e l} * n\right)_{j}}{\sum_{j=1}^{N W S}\left(W I P_{r e l} * n\right)_{j}} * 100
$$

where:

$$
\begin{array}{ll}
\text { OTP }_{\text {rel,j }} & \begin{array}{l}
\text { relative share of a bottleneck situation induced by WIP of } \\
\text { work centre } j[\%]
\end{array} \\
W_{\text {IP }} \text { rel } & \text { relative WIP of work centre } j[\%]
\end{array}
$$

\section{Identifying delivery bottlenecks}

The bottleneck analysis of the $3^{\text {rd }}$ level emphasizes the finding of adequate measures for eliminating bottleneck situations. While the order bottlenecks reveal which work centres have the strongest effect on the achievement of the bottleneck objectives of the $1^{\text {st }}$ level, a combination with the existing potential for improvement is missing still. Regarding mean throughput time and lateness the existing potential is depending on the relative WIP of the work centre because at constant output rate a low WIP level induces a short throughput time. In order to determine that relative WIP level which offers a reasonable compromise between short throughput time and high utilization the theory of Logistic Operating Curves can be employed.

This has been developed at the Institute of Production Systems and Logistics of the University of Hannover (Wiendahl, 1995). The Logistic Operating Curves describe how mean output rate and mean throughput time of a work centre change with the WIP level. On the basis of the Logistic Operating Curve the WIP level can be calculated which offers the best compromise between throughput time and utilization of a work centre. This procedure is called logistic objective trade-off. For this purpose, a target WIP level of the work centre is determined. This figure can be calculated by multiplying mean ideal minimum WIP level with the relative WIP. The ideal minimum WIP describes that WIP level - assuming an ideal process where neither the work centre suffers from a lack of orders nor the orders have to compete for operation at the work centre. Many applications, both, in industrial use and in the development of new methods for PPC, have shown that a general relative WIP level of $250 \%$ is best suited to the logistical targets of each work centre. The calculation of Logistic Operating Curves is extensively covered by Nyhuis and Wiendahl (1999).

According to the significance of the relative WIP it has been found useful to weight the bottleneck analyses of the $2^{\text {nd }}$ level with the relative WIP. Thus those 
work centres can be detected which offer potentialities for WIP reduction. These work centres represent bottlenecks of the $3^{\text {rd }}$ level. The Figures TL $\mathrm{T}_{\text {rel }}$ and $\mathrm{LDD}_{\text {rel }}$ describe the work centres' relative shares of the WIP-induced potentialities for eliminating the bottleneck situation (Windt, 2001):

$$
T L_{r e l, j}=\frac{\left(\operatorname{TTP}_{m} * n * W I P_{r e l}\right)_{j}}{\sum_{j=1}^{N W S}\left(\operatorname{TTP}_{m} * n * W I P_{r e l}\right)_{j}} * 100
$$

where:

$$
\begin{aligned}
& \mathrm{TL}_{\mathrm{rel}, \mathrm{j}} \quad \begin{array}{l}
\text { relative share of WIP-induced potentialities for lead time reduction } \\
\text { of work centre } \mathrm{j}[\%]
\end{array}
\end{aligned}
$$

$$
L D D_{r e l, j}=\frac{\left(\operatorname{LOP}_{m} * n * W I P_{r e l}\right)_{j}}{\sum_{j=1}^{N W S}\left(\operatorname{LOP}_{m} * n * W I P_{r e l}\right)_{j}} * 100
$$

where:

$\mathrm{LDD}_{\text {rel,j }} \quad$ relative share of WIP-induced potentialities for lateness reduction of work centre $\mathrm{j}[\%]$

The bottleneck methodology shown in Figure 4 does not present any combination of the relative WIP with the results of the order throughput bottleneck analysis. This is due to the fact that an order throughput bottleneck would not be eliminated with a reduction in WIP but with an increase in maximum capacity. Besides the calculation of the order throughput bottleneck already comprises the relative WIP.

\section{Identifying production bottlenecks}

The production bottleneck represents the highest hierarchy level in the bottleneck methodology (bottleneck of the $4^{\text {th }}$ level). For this purpose the results from the delivery date bottleneck analysis and the lead time bottleneck analysis are multiplied. Afterwards the relative shares are calculated:

$$
P R_{r e l, j}=\frac{\left(T L_{r e l} * L D D_{r e l}\right)_{j}}{\sum_{j=1}^{N W S}\left(T L_{r e l} * L D D_{r e l}\right)_{j}} * 100
$$

where:

$$
\begin{array}{ll}
\mathrm{PR}_{\mathrm{rel}, \mathrm{j}} & \text { relative degree of achievement of logistical objectives of work } \\
\text { centre } \mathrm{j}[\%]
\end{array}
$$

The additional benefit of this examination lies in the consideration of all relevant key-Figures for bottleneck identification in a combined manner. In the following section the mode of function of the bottleneck methodology is going to be 
presented on the basis of real data from a manufacturer for printed circuit boards cooperating in the production network presented in Figure 2.

\section{APPLICATION AND BENEFIT}

The bottleneck analysis requires feedback data from the job shop. These data should contain details about the single operations of each order, e.g. order time, order sequence, feedback dates. The detailed requisites can be found in (Windt, 2001). Here, we are looking at a factory producing printed circuit boards. The data analyzed contains approximately 3800 orders with 35000 operations and 33 workstations. The data was captured in a period of about three months.
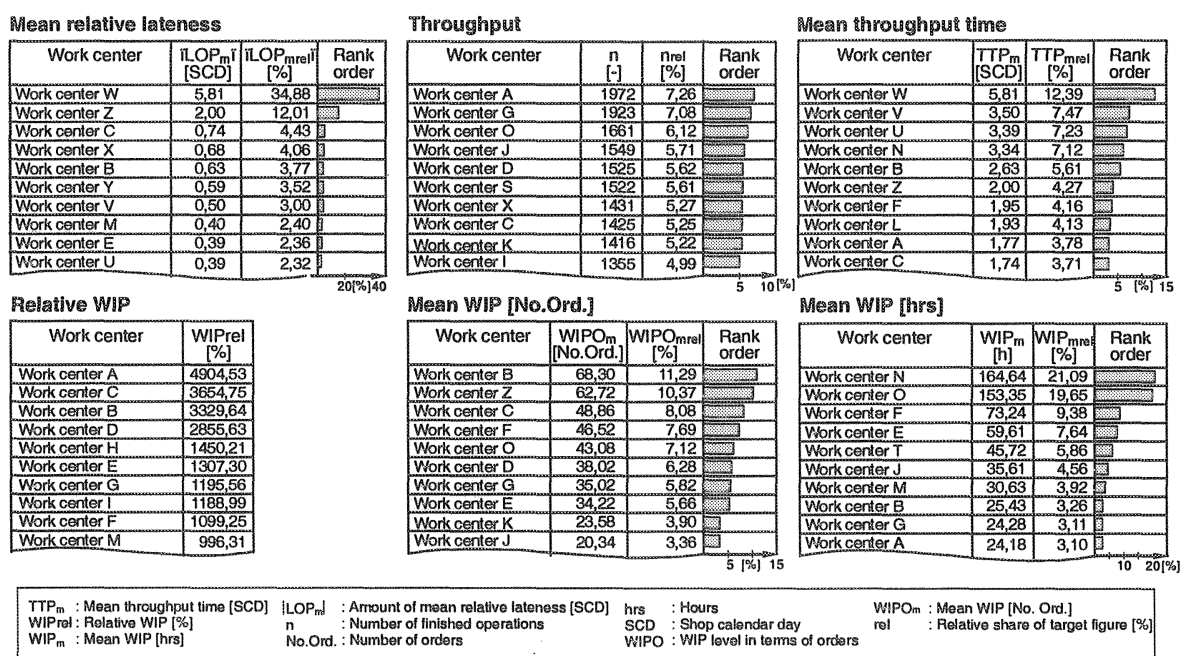

Figure 5-Bottlenecks of the 1st level (resource bottlenecks)

Figure 5 shows the results of the bottleneck analysis of the $1^{\text {st }}$ level. Each table shows the work centres of the analyzed manufacturer regarding different bottleneck criteria is descending order. The analysis of the bottleneck of the $1^{\text {st }}$ level reveals that only one single criterion is employed to detect the bottleneck. Thus, it can happen that work centres are identified as bottlenecks, for instance for the bottleneck criterion of mean throughput time, which only have a strong effect on few orders. Other work centres which may cause shorter throughput times but process a larger number of orders are disregarded although they affect more orders. Regarding for example mean throughput time as the bottleneck criterion, it can be seen that work centre $\mathrm{W}$ represents the bottleneck with 5,81 shop calendar days and a relative share of $12,39 \%$.

The different positions of the same work centre in diverse bottleneck analyses of the $1^{\text {st }}$ level depict that it cannot be concluded for which work centre measures for the elimination of the bottleneck situation should be developed. For this reason the bottleneck analyses of the $2^{\text {nd }}$ and $3^{\text {rd }}$ level have to be applied. Taking into account 
the bottleneck analysis of the $2^{\text {nd }}$ level it can be seen that the work centre $\mathrm{A}$ has taken the first position (Figure 6). Figure 6 points out that work centre A processed a large number of orders. In consequence it has a strong influence on the output of the entire production. Measures for the reduction of the order throughput time should therefore be put on this work centre first and not on the work centre W as it could have been suggested taking into consideration only the results of the bottleneck analysis of the $1^{\text {st }}$ level.

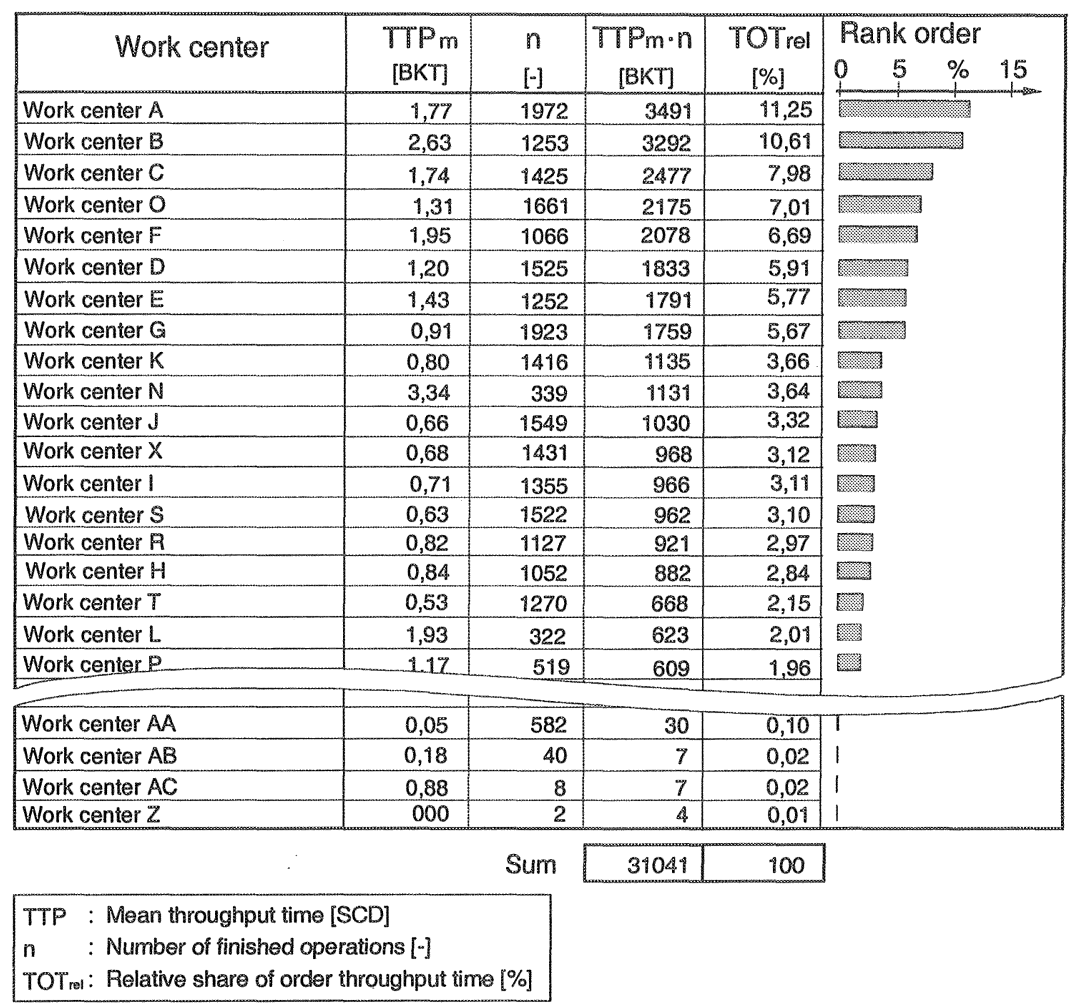

Figure 6 - Bottlenecks of the 2nd level (order bottlenecks)

In the following it is of interest which bottlenecks offer potentialities for WIPinduced reduction of throughput time. Consequently a bottleneck analysis of the $3^{\text {rd }}$ level is carried out. Figure 7 shows the results.

The work centre which offers the highest potential for the reduction of order throughput time can be easily detected. It is work centre A. But this examination does not give any information whether the lead time bottleneck provokes strong impact on the order lateness as well. So the results of the bottleneck analysis of the $3^{\text {td }}$ level are combined for the bottleneck analysis of the $4^{\text {th }}$ level (production bottleneck) in Figure 8.

In order to clarify the results the most relevant work centres are put into a production bottleneck portfolio (Figure 8). The portfolio consists of four quadrants and shows the relative share of WIP-induced potentialities for lead time reduction on 
the $\mathrm{x}$-coordinate and the relative share of WIP-induced potentialities for lateness reduction on the $y$-coordinate. By using the portfolio the work centres can be assigned to four quadrants according to their urgency for measures to be carried out. The portfolio is evidently dominated by three work centres which are located in quadrant 2. These work centres have a high potential for the reduction of order throughput time and a high potential for the reduction of order lateness at the same time.

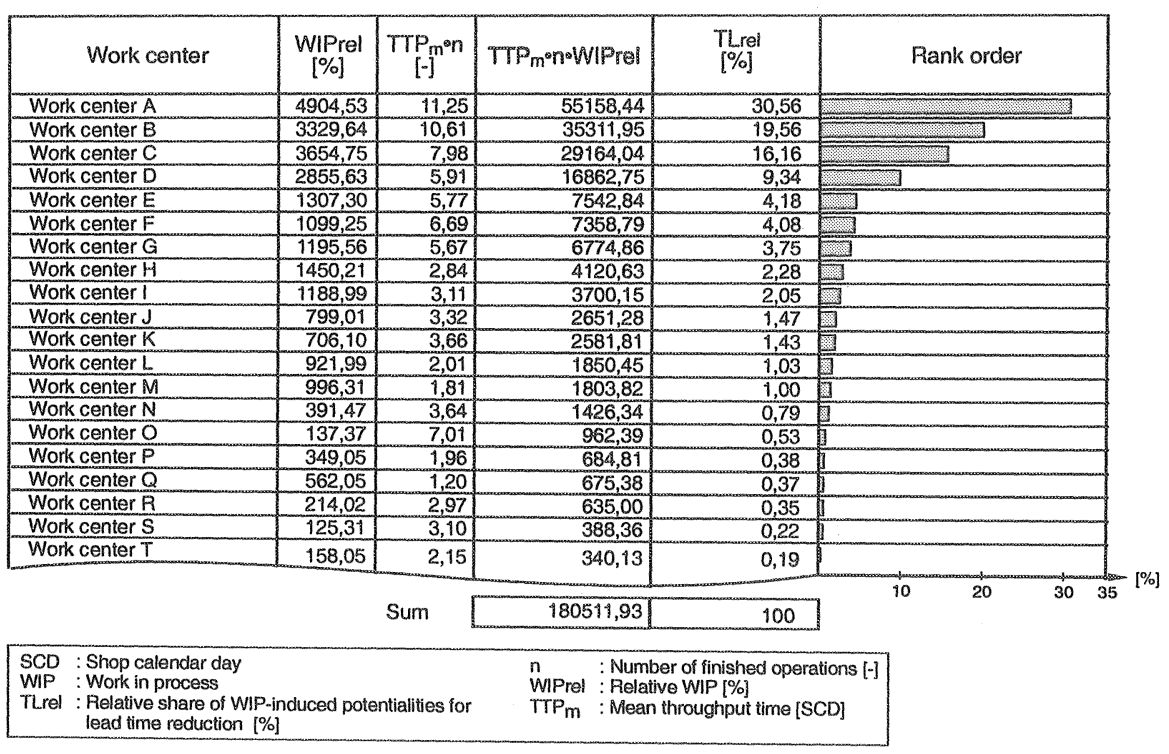

Figure 7-Bottlenecks of the 3rd level (delivery bottlenecks)

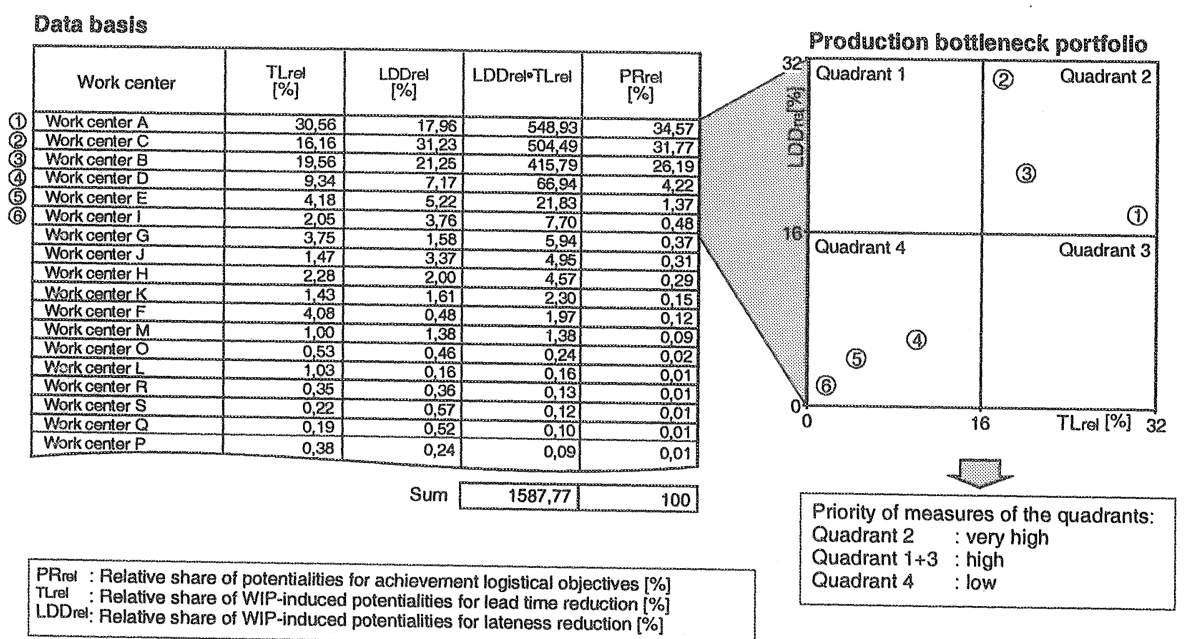

Figure 8 - Bottlenecks of the 4th level (production bottlenecks) 
It can be assumed that a reduction in WIP would result in a maximum benefit for the entire production with respect to the bottleneck criteria. Work centre A has the strongest effect on the achievement of the target Figures in the production. Therefor measures should be put on this work centre first. For the rest of the work centres measures can be rolled out in a subsequent step. The use of the production bottleneck portfolio enables the production manager to find the bottlenecks due to different bottleneck criteria. According to a bottom-down concept he can substantiate the derivation of measures by starting from the production bottleneck and going down to the different levels of bottlenecks.

\section{CONCLUSIONS}

This paper presents a methodology which allows the classification and identification of bottleneck work centres according to different bottleneck criteria. By use of this bottleneck analysis for the production process in production networks, adequate measures for eliminating present bottlenecks, e.g. by subcontracting entire production orders or single processes, and for avoiding future ones, can be derived.

Recent research studies have proven the existence of several bottleneck types according to different logistic performance measures, such as throughput time, schedule reliability, utilization and WIP (work in process) level. For each measure a category of bottleneck types can be defined. Additional bottleneck categories can be derived from a combination of different performance measures. The paper shows how these can be combined together in a bottleneck classification scheme. Applying the methodology in practice, it completes the range of functions of enterprises' monitoring and performance measurement systems. Analyzing the shop floor feed back data of the ERP system, resource bottlenecks can be determined and aggregated to the production bottleneck. The outcomes are usable, concentrated data to manage effectively bottlenecks of enterprises and production networks. The application of the bottleneck-oriented logistic analysis revealed the benefits attainable for companies by using an example of a manufacturer of printed circuits boards.

Production networks and supply chains offer promising opportunities to meet market demands by close cooperation between companies. New production management approaches are required that serve to fully exploit these opportunities. One such opportunity is the collaborative utilization of distributed capacities within a production network. The methodology described in this paper determines those bottleneck resources for whose management collaboration between supply chain partners is required. It is thus a practical as well as essential tool to improve the efficient and effective collaboration of companies. 


\section{REFERENCES}

[1] Dombrowski, U. (1995): Einführung eines standardisierten Multisite-PPS-Systems in Client-Server-Architektur, Tagungsband zum 17. AWF PPS-KongreB "Moderne PPS und Vitale Fabrik", 08.-01. November 1995 in Böblingen.

[2] Forrester, J. W. (1961): Industrial Dynamics, John Wiley \& Sons, New York, USA

[3] Grandori, A.; Soda, G. (1995): Inter-firm Networks: Antecedents, Mechanisms and Forms.

[4] Inger, R.; Braithwaite, A.; Christopher, M. (1995): Creating a manufacturing environment that is in harmony with the market - the "how" of supply chain management. Production Planning \& Control. Vol. 6, No. 3, p. 246 - 257.

[5] Kerner, A. (2001): Modellbasierte Beurteilung der Logistikleistung von Prozessketten. VDI Düsseldorf.

[6] Kernler, H. (1995): Durchlaufzeitverkürzung im PPS-Verbund. ZwF 90 3, p. 95 - 97.

[7] Kuhn, A. (1995): Prozessketten in der Logistik: Entwicklungstrends und Umsetzungsstrategien, in Zusammenarbeit mit: Baron, Chr.; Bernemann, St.; Kaeseler, J.; Manthey, Chr.; Wenzel, S.; Winz, G., Dortmund 1995.

[8] Nassimbeni, G. (1998): Network structures and co-ordination mechanisms - A Texonomy. International Journal of Operations \& Production Management.

[9] Nyhuis, P.; Wiendahl, H.-P. (1999): Logistische Kennlinien. Grundlagen, Werkzeuge und Anwendungen. Springer, Berlin.

[10] Semich, W. (1994): Information replaces Inventory at the virtuell Corporation. Datamation.

[11] Spur, G. (1996): Informations-und Kommunikationstechnik in der Produktion, ZwF 91, p. $62-63$.

[12] Starr, M.K. (1991): Global Corporate Alliances and the Competitive Edge. Quorum Books, New York.

[13] Wiendahl, H.-P. (1995): Load-oriented Manufacturing Control. Berlin, Heidelberg: Springer-Verlag.

[14] Wiendahl, H.-P.; Helms, K. (1998): Successful Operating in an ,Alliance of the Best". Organizing the Extended Enterprise (ed. P. Schönsleben, A. Büchel), Champman \& Hall, London.

[15] Wiendahl, H.-P.; Helms, K.; Höbig, M. (1998a): Management of Variable Production Networks, Annals of the CIRP.

[16] Windt, K. (2001): Engpaßorientierte Fremdvergabe in Produktionsnetzen. VDI, Düsseldorf. 